\title{
The usefulness of echocardiography in the diagnosis of myocarditis in a young male after sudden cardiac arrest
}

\author{
Wioletta Sacharczuk ${ }^{1}$, Rafał Dankowski ${ }^{1}$, and Andrzej Szyszka ${ }^{2}$ \\ ${ }^{1}$ Poznan University of Medical Sciences \\ ${ }^{2}$ Affiliation not available
}

January 21, 2021

\begin{abstract}
The diagnosis of acute myocarditis (AM) remains challenging because of its diverse clinical manifestations. Thus, a wide range of diagnostic tests may be warranted. Although cardiac magnetic resonance (CMR) is the preferred imaging technique, it may not be applicable in the acute AM phase. Our case report highlights the usefulness and diagnostic accuracy of echocardiographic examination. In the first 2-dimensional echocardiography, the focal echobright was presented. A reduced value of global longitudinal strain, and regional disturbances of segmental myocardial strain, both longitudinal and circumferential, in the epicardial layer, were detected with a good correlation with CMR results.
\end{abstract}

Title: The usefulness of echocardiography in the diagnosis of myocarditis in a young male after sudden cardiac arrest

Running head: Echocardiography in the diagnosis of myocarditis

Authors: Wioletta Sacharczuk, MD, PhD ${ }^{1}$, Rafał Dankowski, MD, PhD ${ }^{1}$, Andrzej Szyszka MD, PhD, prof. ${ }^{1}$

Institution. ${ }^{1}$ Second Department of Cardiology, Poznan University of Medical Sciences, Poznan, Poland

Corresponding author. Wioletta Sacharczuk. Telephone number: $+48+48666940$ 480, fax number:, e-mail address:wioletta.sacharczuk@wp.pl

Abstract: The diagnosis of acute myocarditis (AM) remains challenging because of its diverse clinical manifestations. Thus, a wide range of diagnostic tests may be warranted. Although cardiac magnetic resonance (CMR) is the preferred imaging technique, it may not be applicable in the acute AM phase. Our case report highlights the usefulness and diagnostic accuracy of echocardiographic examination. In the first 2dimensional echocardiography, the focal echobright was presented. A reduced value of global longitudinal strain, and regional disturbances of segmental myocardial strain, both longitudinal and circumferential, in the epicardial layer, were detected with a good correlation with CMR results.

Introduction. A variety of clinical scenarios makes the diagnosis of myocarditis a clinical challenge ${ }^{1}$. Among the various imaging techniques, echocardiography plays an important role in the diagnostic process ${ }^{2}$, and the analysis of myocardial strain may be helpful ${ }^{3}$. In this case report, we highlight the usefulness of echocardiography in the diagnosis of myocarditis in a young male after sudden cardiac arrest.

Case description. A 30-year-old patient was admitted to the intensive care unit after resuscitated sudden cardiac arrest. He lost his consciousness during an amateur sports competition (a 10-kilometer run). The patient did not suffer from any chronic diseases. A few days before the competition he had a cold, but nevertheless decided to take part in the run. The electrocardiogram on admission revealed Q waves in I, II, aVF, and in V4 to V6 leads. High-sensitive troponin T and D-dimer levels were increased $(2577 \mathrm{pg} / \mathrm{ml}$ 
(upper reference level: $14 \mathrm{pg} / \mathrm{ml}$ ) and $2836 \mathrm{ng} / \mathrm{ml}$ (upper reference level: $500 \mathrm{ng} / \mathrm{ml}$, respectively). The inflammatory markers were also above the normal range; white blood cells (WBC) were $14 \times 10^{9} / \mathrm{L}$ and high-sensitive C-reactive protein (hs-CRP) was $78 \mathrm{mg} /$ l. Point-of-care echocardiography showed hypokinesis of the apex; the left ventricular ejection fraction (LVEF) was $52 \%$, and no other pathologies were reported. Both coronary angiography and pulmonary angiogram were negative. A full echocardiographic examination repeated on day 7 of hospitalization showed new wall motion abnormalities including akinesis of the apex, hypokinesis of the apical and mid segments of the anterior, posterior and lateral walls, and the apical, mid, and basal segments of the inferior wall. In these regions, the focal echobright sign was also observed (fig.1). The LVEF was slightly decreased (48\%). Analysis of myocardial strain parameters revealed decreased global function of the LV; the global longitudinal strain (GLS) was - 14.4\%. Further detailed of-line analysis revealed normal GLS values in the endocardial layer ( $-16.6 \%)$ and decreased values in the epicardial layer ($12.7 \%$ ) (fig.2). Layer-specific global circumferential strain (GCS) values were $-22.3 \%$ for the endocardium and $-9.6 \%$ for the epicardium. Analysis of regional strain also showed local disturbances of both longitudinal and circumferential parameters. In the epicardial layer, reduced values of the longitudinal strain were observed in the posterior and lateral walls, in the apical segments of the inferior and anterior walls, and in the apex (fig.2). In the endocardial layer, deterioration was observed in the basal and mid segments of the posterior wall only. Analysis of circumferential strain at the epicardial layer showed decreased values of the apex, and the inferior, posterior and lateral walls (fig.3). Cardiac magnetic resonance (CMR) examination showed edema and hyperemia in the posterior and lateral wall. According to the Lake Louise criteria (LLc), myocarditis was diagnosed ${ }^{4}$. After full recovery, the patient was discharged home with advice to postpone physical activity for at least 3 months.

A follow-up examination was conducted after 6 months. Echocardiography showed persistent akinetic basal segments of the inferior wall and hypokinetic apical segments of the lateral and anterior walls. The LVEF was $57 \%$, average GLS $-18.6 \%$. The GLS values in the endo- and epicardial layers were found to have improved (-20.8\% and $-16.8 \%$ respectively, fig.4). Global circumferential strain values improved in the endocardial layer but were persistently decreased in the epicardial layer in the basal segments of the posterior and inferior walls (-29.3\% and $-13.6 \%$ respectively; fig.5). CMR revealed normal systolic function (LVEF $55 \%$ ), and reduced thickness of the basal segments of the inferior and posterior regions, with high suspicion of necrosis (late post-gadolinium myocardial enhancement).

Discussion. In this case report, we showed an additional benefit of 2-dimensional echocardiography in the diagnosis of myocarditis. Firstly, the focal echobright sign corresponded to the regional left ventricle contractility disturbances (fig.1). It has been reported in the literature that the focal echobright is distinctive to the acute phase of myocarditis only ${ }^{1}$. Indeed, at the 6-month follow-up, we did not see this phenomenon any more. Furthermore, we found that the detection of a reduced GLS value, regional disturbances of segmental myocardial strain, and diminished values of both GLS and GCS in the epicardial layer, could help to diagnose myocarditis. The inflammatory process in acute myocarditis is located mostly in the epicardial layer and mainly in the lateral wall ${ }^{5}$. Thus, the layer-specific analysis of myocardial deformation could be of value in the diagnostic process ${ }^{6}$. Myocardial strain disturbances were confirmed by CMR, which currently plays a major role in the diagnostic pathway of myocarditis ${ }^{7}$. It has been shown that the assessment of myocardial strain could be of value in the diagnosis of myocarditis ${ }^{8}$. More recently, a good correlation between global and regional strain and CMR has been demonstrated ${ }^{9}$. In our patient, we found decreased values of both GLS and GCS, mainly in the epicardial layer. Diminished GCS was also observed at the follow-up and corresponded to the CMR results. It has been shown that the circumferential strain, in contrast to the longitudinal strain, is more sensitive to distinguish scar transmurality ${ }^{10}$.

Our case study shows that echocardiography offers several techniques and modalities which could be very helpful in the early diagnosis of myocarditis. Although CMR is the preferred imaging technique, it may not be applicable in the acute phase of the disease. The finding is very important nowadays, during the COVID-19 pandemic, when the availability of some procedures may be limited. Thus, the coexistence of the echobright and myocardial strain abnormalities, especially in the epicardial layer, may be very useful in the diagnosis of myocarditis. 
Refrences:

1. Saricam E, Saglam Y, Hazirolan T. Clinical evaluation of myocardial involvement in acute myopericarditis in young adults. BMC Cardiovasc Disord . 2017;17(1):129-129. doi:10.1186/s12872-017-0564-8

2. Caforio ALP, Pankuweit S, Arbustini E, et al. Current state of knowledge on aetiology, diagnosis, management, and therapy of myocarditis: a position statement of the European Society of Cardiology Working Group on Myocardial and Pericardial Diseases. Eur Heart J . 2013;34(33):2636-2648. doi:10.1093/eurheartj/eht210

3. Escher F, Kasner M, Kühl U, et al. New echocardiographic findings correlate with intramyocardial inflammation in endomyocardial biopsies of patients with acute myocarditis and inflammatory cardiomyopathy.Mediators Inflamm . 2013;2013:875420. doi:10.1155/2013/875420

4. Ferreira VM, Schulz-Menger J, Holmvang G, et al. Cardiovascular Magnetic Resonance in Nonischemic Myocardial Inflammation. J Am Coll Cardiol . 2018;72(24):3158-3176. doi:10.1016/j.jacc.2018.09.072

5. Mahrholdt H, Wagner A, Deluigi CC, et al. Presentation, Patterns of Myocardial Damage, and Clinical Course of Viral Myocarditis.Circulation . 2006;114(15):1581-1590. doi:10.1161/CIRCULATIONAHA.105.606509

6. Caspar T, Germain P, Ghannudi SE, et al. Acute Myocarditis Diagnosed by Layer-Specific 2D Longitudinal Speckle Tracking Analysis.Echocardiography . 2016;33(1):157-158. doi:10.1111/echo.13045

7. Friedrich MG, Sechtem U, Schulz-Menger J, et al. Cardiovascular Magnetic Resonance in Myocarditis: A JACC White Paper. J Am Coll Cardiol . 2009;53(17):1475-1487. doi:10.1016/j.jacc.2009.02.007

8. Hsiao J-F, Koshino Y, Bonnichsen CR, et al. Speckle tracking echocardiography in acute myocarditis. Int J Cardiovasc Imaging . 2013;29(2):275-284. doi:10.1007/s10554-012-0085-6

9. Leitman M, Vered Z, Tyomkin V, et al. Speckle tracking imaging in inflammatory heart diseases. Int $J$ Cardiovasc Imaging . Published online November 27, 2017. doi:10.1007/s10554-017-1284-y

10. Geyer H, Caracciolo G, Abe H, et al. Assessment of Myocardial Mechanics Using Speckle Tracking Echocardiography: Fundamentals and Clinical Applications. J Am Soc Echocardiogr . 2010;23(4):351-369. doi:10.1016/j.echo.2010.02.015 


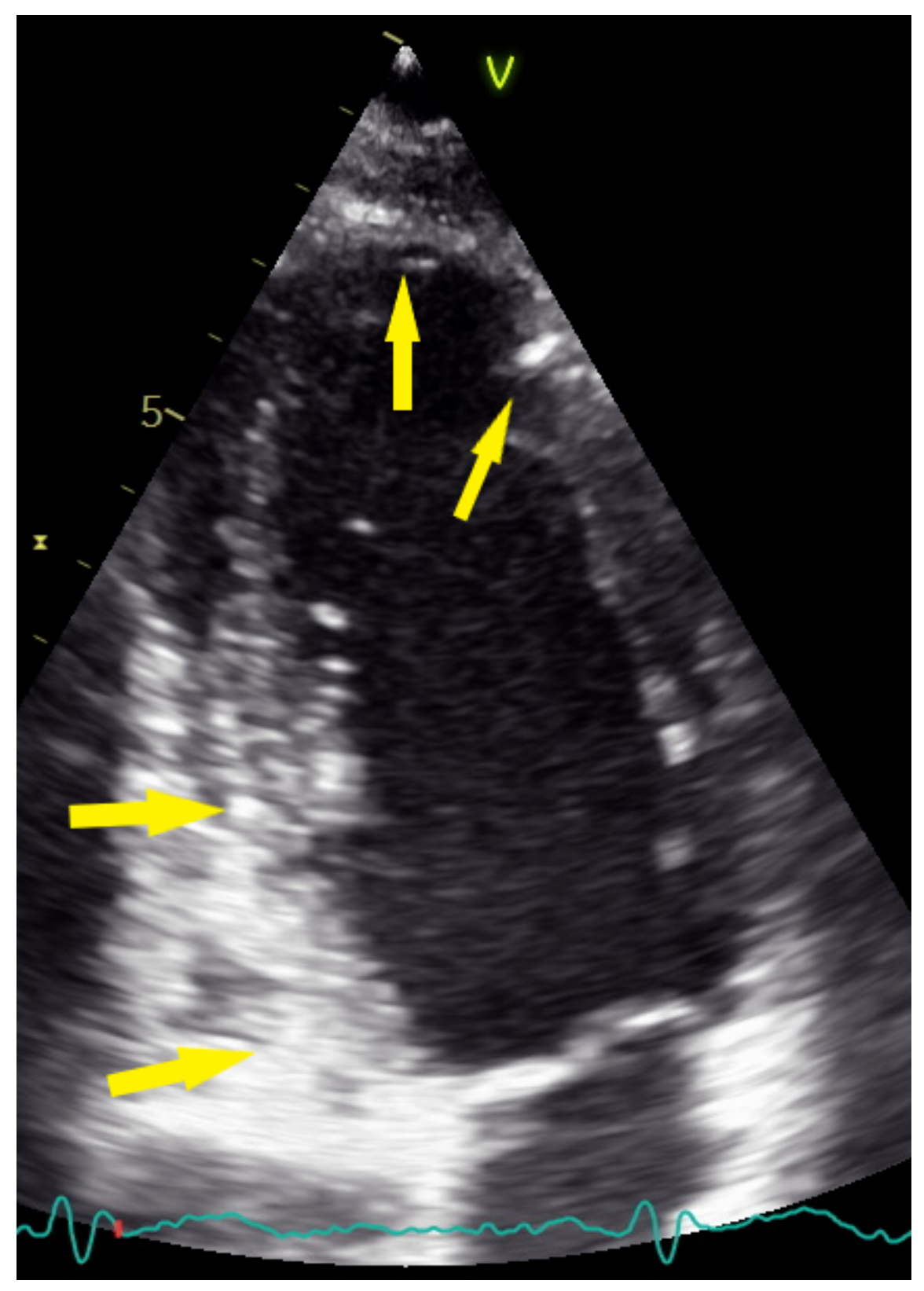



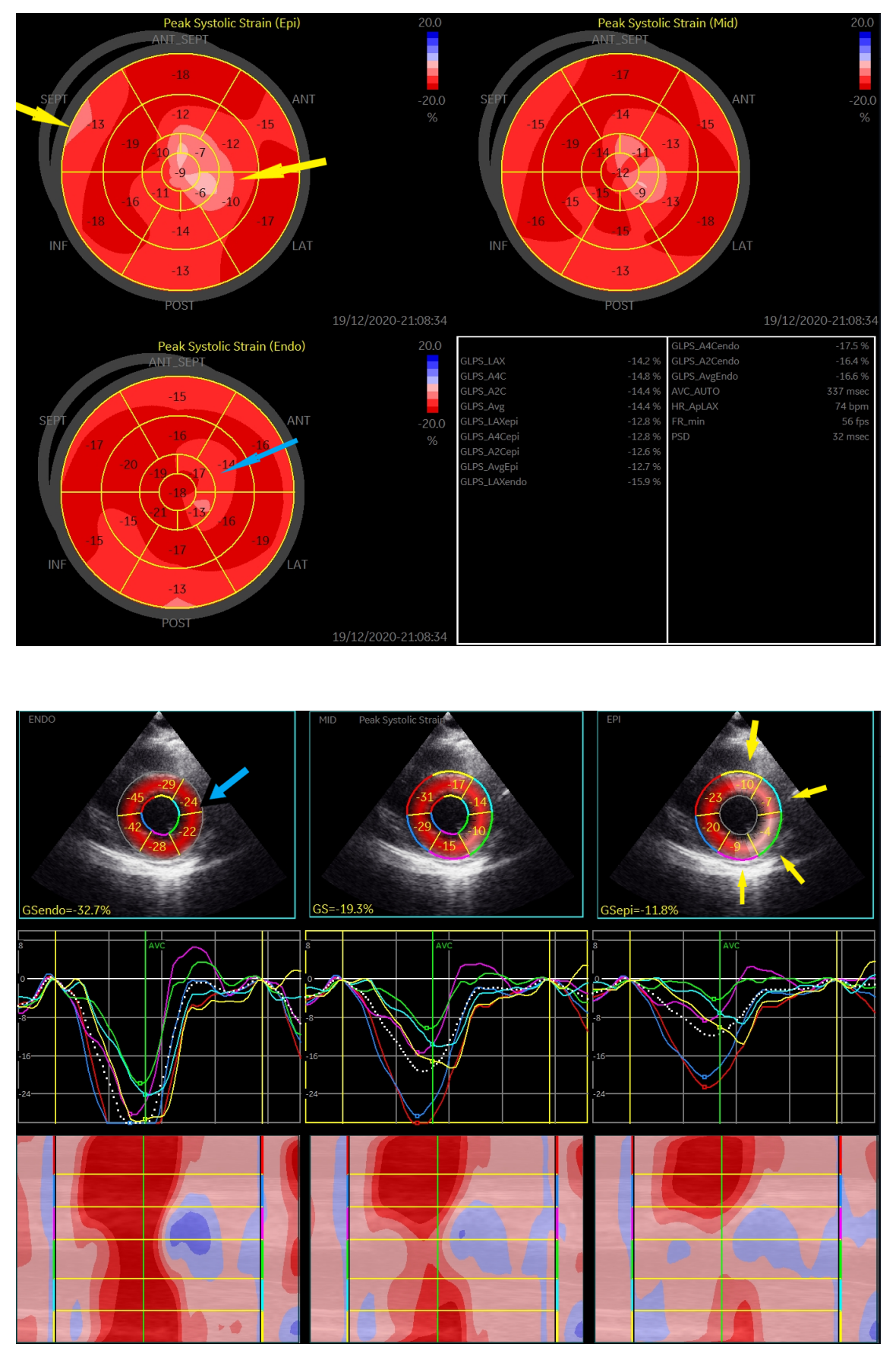

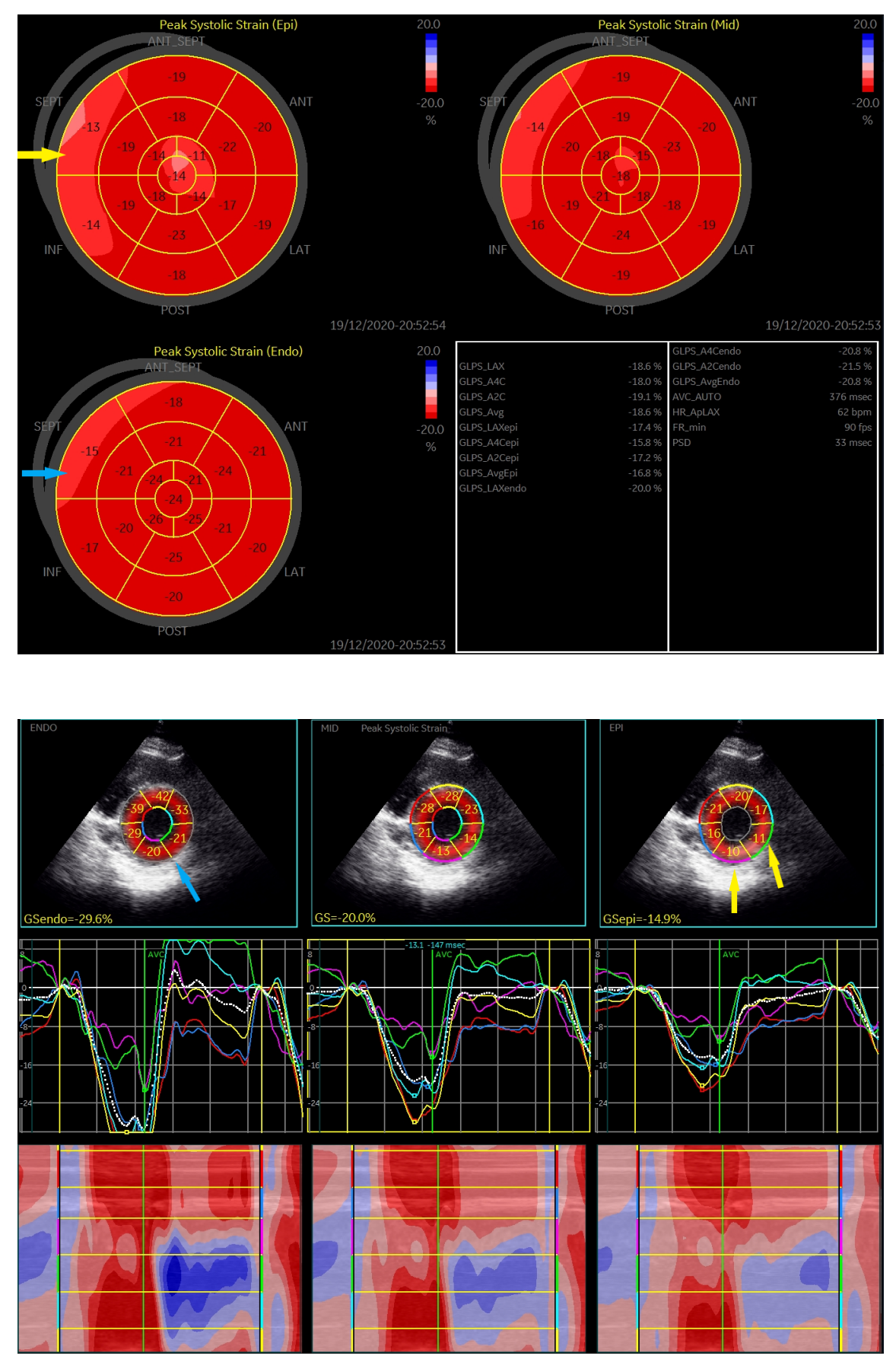